\title{
Assessment of Cardiovascular Autonomic Functions to Predict Development of Pregnancy Induced Hypertension
}

\author{
Nandini Kapoor', Rajeev Sharma', Munish Ashat', Anju Huria'2, Ghansham Mishra ${ }^{3}$ \\ Dept. of Physiology', Dept. Obs/Gyn², Govt. Medical College and Hospital, Chandigarh, Dept. of SPM³, \\ GGS Medical College, Faridkot (Punjab)
}

\begin{abstract}
Aim: This study aimed at performing autonomic function tests to predict $\mathrm{PIH}$ at early stage of pregnancy.

Methods: 200 pregnant women between 12 to 20 weeks of gestation were selected for study. Handgrip dynamometer test (HGT) and Cold pressor test (CPT) were performed to assess sympathetic cardiovascular functions.

Result: It was observed that 31 women out of 200 pregnant women developed PIH subsequently. ROC curve analysis was done to find out cut off values of high sensitivity and specificity for rise in systolic blood pressure $(\triangle \mathrm{SBP})$ and diastolic blood pressure $(\triangle \mathrm{DBP})$ during CPT and HGT. It was observed that out of 31 women who developed $\mathrm{PIH} / \mathrm{PE}, 87.1 \%$ had $\triangle \mathrm{SBP} \geq 14 \mathrm{~mm} \mathrm{Hg}$ (cut off value) and $83.9 \%$ had $\triangle \mathrm{DBP} \geq 10 \mathrm{~mm} \mathrm{Hg}$ (cut off value) during CPT, both of which were statistically significant $(p<0.001)$. Similarly for HGT, 31 women who developed PIH, $\triangle \mathrm{SBP} \geq 16 \mathrm{~mm} \mathrm{Hg}$ and $\triangle \mathrm{DBP} \geq 14 \mathrm{~mm} \mathrm{Hg}$ (cut off values) was observed in $96.8 \%$ and $83.9 \%$ women respectively $(p<0.001)$. Discriminant analysis showed that the reliability of both the tests in predicting $\mathrm{PIH}$ was very high $(\mathrm{p}<0.001)$.
\end{abstract}

Conclusion: Thus it can be concluded from the study that heightened blood pressure response to physiological stimuli like cold and isometric exercise early in pregnancy could be used as a suitable tool to predict development of $\mathrm{PIH}$.

Key words: Cold pressor test, early detection, Handgrip dynamometer test, $\mathrm{PIH}$

\section{Introduction}

Pregnancy induced hypertension $(\mathrm{PIH})$ is a syndrome that arises in pregnancy and is diagnosed by presence of hypertension (blood pressure of $140 / 90 \mathrm{~mm} \mathrm{Hg}$ or more for the first time in pregnancy and on two separate occasions) first detected after 20 weeks of gestation $\mathrm{PIH} /$ Pre eclampsia is one of the most common cause of both maternal and neonatal morbidity \& mortality affecting $5 \%$ to $8 \%$ of all pregnancies. The incidence of maternal deaths that are associated with this disorder has been found to be $9.1 \%$ (Asia), 16.1\% (developed countries) and $25.7 \%$ (Latin America) ${ }^{1-3}$.

Pregnancy is associated with profound adaptive changes in the maternal hemodynamics. Autonomic nervous system plays a central role in the adaptation

Correspondence

Dr. Rajeev Sharma MD

Associate Prof. Dept of Physiology

Guru Gobind Singh Medical College, Faridkot (Punjab)

Phone: 09815618171

Email : rajeevsharma.md@gmail.com of the cardiovascular system to various needs of pregnancy. Impairment of autonomic functions has been suggested as one of the cause of PIH. There have been reports of greater resting sympathetic output in cases of PIH as compared to normal pregnancy ${ }^{4}$. The central sympathetic output has also been found to be increased to a much greater extent in women with PIH as compared to normal pregnant women ${ }^{5}$. In some other studies, increased sympathetic activity as well as decreased vagal tone has been found to be associated with $\mathrm{PIH} / \mathrm{PE}^{6,7}$.

Although the disorder usually becomes apparent only in the third trimester of pregnancy, evidence is available that underlying pathophysiological abnormalities appear early in the pregnancy ${ }^{8}$. There 
have been attempts for early prediction of PIH/PE by using a variety of biological, biochemical and biophysical markers implicated in the pathophysiology of Preeclampsia. But early prediction is still insufficient in clinical practice ${ }^{9,10}$. Therefore early detection of high risk patients is essential for prophylactic interventions to reduce morbidity \& mortality associated with this syndrome. Evaluation of circulatory alterations during sustained isometric contraction or exposure to cold stimulus is a useful tool to assess cardiac functions. Hence this study was planned to ascertain whether Cold pressor test and Handgrip test performed early in pregnancy could be useful predictors of PIH.

\section{Methods}

The study was conducted in Department of Physiology and Department of Obstetrics and Gynecology at Government Medical College and Hospital, Chandigarh. A total of 200 pregnant women from antenatal clinic with gestational period between 12 to 20 weeks were included in the study after approval of the protocol by institute's ethics committee. All subjects were explained of the procedure to be undertaken and a written consent was obtained as per Helsinki Declaration, modified according to test protocol.

Patients with Chronic hypertension, diabetes, chronic renal disease, Heart ailments and chronic respiratory illness were not included in the study.

The subjects were made to rest for 10 minutes before recording their base line systolic and diastolic blood pressure in sitting posture, as per standard procedures. Two tests i.e. Isometric hand dynamometer test and Cold pressor test were performed to assess Sympathetic Cardiovascular functions. A gap of 30 minutes was observed in between performing two tests

\section{Hand grip Dynamometer Test (HGT)}

The subjects were explained about the procedure through self demonstration by investigators.

The subjects were asked to perform Maximum Voluntary Contraction (MVC) by gripping the handgrip dynamometer, as hard as possible for few seconds and maximum force exerted was noted down. After giving rest for few minutes the subjects were made to perform isometric exercise at $30 \%$ of maximal voluntary contraction by gripping the hand dynamometer for 3-5 minutes or till the subject could sustain the effort comfortably. Systolic and diastolic blood pressure was recorded at interval of one minute during the period of exercise and rise in Systolic Blood Pressure $(\triangle \mathrm{SBP})$ and Diastolic Blood Pressure $(\triangle \mathrm{DBP})$ were noted. The response was considered as the difference between maximum BP obtained during exercise and the resting BP

\section{Cold Pressor Test}

The right hand of the subject was immersed up to the wrist in cold water at temperature of $4-6^{\circ} \mathrm{C}$ for 1 minute. Blood pressure was recorded at 1 minute of immersion of hand. After taking out the hand, blood pressure was measured after every minute, till it returned to baseline.

The increase in systolic and diastolic blood pressure from the baseline value, known as response was obtained.

The subjects were then followed up and examined at the time of delivery. They were considered to have developed PIH if their blood pressure was $>140 / 90$ $\mathrm{mmHg}$ on two separate occasions minimum of six hours apart with or without proteinuria.

\section{Statistical Analysis}

Statistical analysis was done by using SPSS-10. Data pertaining to change in blood pressure during the tests were compared by using Wilcoxon Signed Rank Test. Receiver Operating Characteristics curve (ROC curve) analysis was performed to know the cut off values of high sensitivity and specificity for change in systolic and diastolic blood pressure during the two tests. Fisher Exact Test was applied to analyse the number of subjects who had blood pressure increase above the cut off value and developed PIH subsequently. Discriminant analysis was performed to get the discriminant function (regression model) for predicting the development of PIH based on change in blood pressure during both the tests.

\section{Results}

In the study group of 200 women, 31 women developed $\mathrm{PIH}$ where as rest 169 remained normal $(\mathrm{N})$ during the course of pregnancy. Resting systolic blood pressure value in the study group was found to be $114 \pm 8.20$ $\mathrm{mmHg}$ while resting diastolic blood pressure value was $73.18 \pm 7.20 \mathrm{mmHg}$. Table 1 depicts the mean \pm SD increase in systolic blood pressure $(\triangle \mathrm{SBP})$ and diastolic blood pressure $(\triangle \mathrm{DBP})$ during Cold Pressor Test (CPT) and Hand Grip Dynamometer Test (HGT) in all these subjects respectively. The increase in blood pressure $(\triangle \mathrm{SBP}$ and $\triangle \mathrm{DBP}$ ) during both the tests i.e. CPT and HGT, was found to be more in pregnant women who subsequently developed PIH as compared to women with normal outcome and was statistically significant $(\mathrm{P}<0.001)$ on applying Wilcoxon Signed Rank test. 
Table 1. Mean \pm sd change in blood pressure during cold pressor and handgrip test

\begin{tabular}{|c|c|c|c|c|}
\hline Test & $\begin{array}{l}\text { Blood Pressure } \\
(\mathrm{mm} \mathrm{Hg})\end{array}$ & $\begin{array}{l}\text { Normal } \\
n=169\end{array}$ & $\begin{array}{l}\text { PIH } \\
n=31\end{array}$ & $\begin{array}{l}\text { p Value } \\
\text { (Asymp. Sig. } 2 \text {-tailed)* }\end{array}$ \\
\hline \multirow{2}{*}{$\begin{array}{l}\text { Cold Pressor Test } \\
\text { (CPT) }\end{array}$} & $\Delta \mathrm{SBP}(\mathrm{Mean} \pm \mathrm{SD})$ & $12.31 \pm 2.82$ & $18.45 \pm 3.33$ & $<0.0001$ \\
\hline & $\triangle \mathrm{DBP}(\mathrm{Mean} \pm \mathrm{SD})$ & $6.90 \pm 1.98$ & $14.77 \pm 5.13$ & $<0.0001$ \\
\hline \multirow[t]{2}{*}{$\begin{array}{l}\text { Hand Grip Test } \\
\text { (HGT) }\end{array}$} & $\Delta \mathrm{SBP}(\mathrm{Mean} \pm \mathrm{SD})$ & $13.71 \pm 2.81$ & $24.90 \pm 5.74$ & $<0.0001$ \\
\hline & $\Delta \mathrm{DBP}($ Mean $\pm \mathrm{SD})$ & $10.92 \pm 3.1$ & $19.22 \pm 5.81$ & $<0.0001$ \\
\hline
\end{tabular}

$*$ p value $<0.001$ is significant

Receiver operating characteristics curve (ROC curve) analysis was performed to identify the women more prone to develop PIH and a cut off value of $14 \mathrm{~mm}$ $\mathrm{Hg}$ for increase in systolic blood pressure $(\triangle \mathrm{SBP})$ and $10 \mathrm{~mm} \mathrm{Hg}$ for increase in diastolic blood pressure ( $\triangle \mathrm{DBP})$ was selected for the Cold pressor test as these values were found to have high sensitivity and specificity.
Out of 31 women who developed PIH, $\triangle \mathrm{SBP}$ of $\geq 14$ $\mathrm{mm} \mathrm{Hg}$ (cut off value) was observed in 27 women $(87.1 \%)$ and $\triangle \mathrm{DBP}$ of $\geq 10 \mathrm{~mm} \mathrm{Hg}$ was observed in 26 women $(83.9 \%)$ which was statistically significant $(\mathrm{P}<0.001)$ using Fisher's Exact test (Table 2).

Similarly, ROC curve analysis was performed for HGT and a cut off value of high specificity and sensitivity was found to be $16 \mathrm{~mm} \mathrm{Hg}$ for increase in systolic blood pressure $(\triangle \mathrm{SBP})$ and $14 \mathrm{~mm} \mathrm{Hg}$ for increase

Table 2. Number (\%) of patients with $\Delta$ SBP and $\Delta$ DBP values above and below cut off value during cold pressor test (CPT) and handgrip test (HGT) $n=200$

\begin{tabular}{|c|c|c|c|c|}
\hline \multirow{6}{*}{$\begin{array}{l}\text { Cold Pressor Test } \\
\text { (CPT) }\end{array}$} & & \multirow[t]{2}{*}{$\begin{array}{l}\text { Blood Pressure } \\
(\mathrm{mm} \mathrm{Hg})\end{array}$} & \multicolumn{2}{|c|}{$\begin{array}{l}\text { Outcome of } \\
\text { Pregnancy }\end{array}$} \\
\hline & & & $\begin{array}{l}\text { Normal } \\
(n=169)\end{array}$ & $\begin{array}{l}\text { PIH } \\
(\mathrm{n}=31)\end{array}$ \\
\hline & \multirow{2}{*}{$\Delta \mathbf{S B P}$} & Increase $<14$ & $146(86.4 \%)$ & $4(12.9 \%)$ \\
\hline & & Increase $\geq 14$ & $23(13.6 \%)$ & $27(87.1 \%)$ \\
\hline & \multirow{2}{*}{$\Delta \mathbf{D B P}$} & Increase $<10$ & $164(97 \%)$ & $5(16.1 \%)$ \\
\hline & & Increase $\geq 10$ & $5(3.0 \%)$ & $26(83.9 \%$ \\
\hline \multirow{4}{*}{$\begin{array}{l}\text { Handgrip } \\
\text { Dynamometer Test } \\
\text { (HGT) }\end{array}$} & \multirow{2}{*}{$\Delta \mathbf{S B P}$} & Increase $<16$ & $156(92.3 \%)$ & $1(3.2 \%)$ \\
\hline & & Increase $\geq 16$ & $13(7.7 \%)$ & $30(96.8 \%)$ \\
\hline & & Increase $<14$ & $156(92.3 \%)$ & $5(16.1 \%)$ \\
\hline & $\triangle \mathbf{D B F}$ & Increase $\geq 14$ & $13(7.7 \%)$ & $26(83.9 \%)$ \\
\hline
\end{tabular}


Table 3. Parameters of discriminant analysis in cold pressor test (CPT) and hand grip test (HGT) $n=200$

\begin{tabular}{llllll}
\hline Tests & $\begin{array}{l}\text { Blood } \\
\text { Pressure } \\
(\mathbf{m m} \mathbf{H g})\end{array}$ & $\begin{array}{l}\text { Canonical discriminant } \\
\text { function }\end{array}$ & Centroid & p-value & Reliability \\
\hline CPT & $\Delta$ SBP & $\mathrm{y}=-4.719+0.309 * \Delta \mathrm{SBP}$ & $-0.963,0.963$ & 0.000 & $84 \%$ \\
& $\Delta \mathrm{DBP}$ & $\mathrm{y}=-3.005+0.264 * \Delta \mathrm{DBP}$ & $-0.875,0.875$ & 0.000 & $88 \%$ \\
$\mathbf{H G T}$ & $\Delta \mathrm{SBP}$ & $\mathrm{y}=-4.858+0.242 * \Delta \mathrm{SBP}$ & $-1.335,1.335$ & 0.000 & $92 \%$ \\
& & $\mathrm{y}=-3.359+0.222 * \Delta \mathrm{DBP}$ & $-1.037,1.037$ & 0.000 & $90 \%$ \\
\hline
\end{tabular}

in diastolic blood pressure $(\triangle \mathrm{DBP})$. For HGT also, statistically significant number of women $(p<0.001)$ had their blood pressure increase $(\triangle \mathrm{SBP}$ and $\triangle \mathrm{DBP})$ to more than cut off values (Table 2)

Canonical discriminant function was obtained by performing discriminant analysis for $\triangle \mathrm{SBP}$ and $\triangle \mathrm{DBP}$ values of Hand grip test as well as Cold pressor test (Table 3). To perform this analysis, we classified the subjects into two groups, one who did not develop $\mathrm{PIH}$ and second who developed PIH during pregnancy. From this analysis it was found that discriminant function has correctly classified $92 \%$ of cases in terms of $\triangle \mathrm{SBP}$ values and $90 \%$ of cases in terms of $\triangle \mathrm{DBP}$ values of Hand Grip test. Similarly discriminant function has correctly classified $84 \%$ of cases in terms of $\triangle \mathrm{SBP}$ values and $88 \%$ of cases in terms of $\triangle \mathrm{DBP}$ values of Cold Pressor Test. This shows that discriminant function has good discriminatory power and the reliability of the tests was high. Significance of discriminant function was further confirmed by Chi square test (Wilk's Lambda) and the p value was found to be $<0.001$ (highly significant).

\section{Discussion}

The association between alteration in autonomic cardiovascular control and development of hypertension in pregnancy has been investigated for some time. Use of non invasive methods for assessment of autonomic functions has been an area of special interest since they have the advantage of having minimal risk for the mother and the fetus ${ }^{6}$.

In the present study we used two autonomic function tests viz Cold Pressor Test and Handgrip Test for early prediction of the development of PIH. Results revealed that women who developed PIH subsequently had heightened response to cold pressor test in the form of more increase in systolic as well as diastolic blood pressure as compared to healthy pregnant women who did not develop PIH. This was evident by the fact that $87 \%$ of the women who developed PIH had $\triangle \mathrm{SBP}$ of more than $14 \mathrm{~mm}$ of $\mathrm{Hg}$ (cut off value) and $83 \%$ of women had $\triangle \mathrm{DBP}$ of more than $10 \mathrm{~mm} \mathrm{Hg}$ (cut off value).Our results are in line with the results of Woisetschlager ${ }^{9}$ et al who observed more pronounced increase in systolic as well as diastolic blood pressure in response to Cold pressor test in women who developed PIH later and attributed this increase to increased vasoconstrictive response to physiological stimulus (Cold in this case).

Similar type of heightened response in $\triangle \mathrm{SBP} \& \triangle \mathrm{DBP}$ was observed during handgrip dynamometer test also in case of women who developed PIH later. Handgrip test conducted by Tomoda et al for predicting PIH have shown more increase in $\triangle \mathrm{SBP}$ during isometric exercise in pregnant women who develop PIH later. They have also shown this test to have high sensitivity in predicting $\mathrm{PIH}^{10}$. The results in our study show that probability of development of PIH in the pregnant women with blood pressure values higher than cut off values was very high.

Degani ${ }^{11}$ et al have found the sensitivity and specificity of Handgrip test for predicting the PIH to be $81 \%$ and $96.5 \%$ respectively. Baker ${ }^{12}$ et al have also demonstrated sensitivity of $80 \%$ and Specificity of $92 \%$ of Handgrip test in predicting PIH and preeclampsia. These observations in our results could be attributed to increased vascular reactivity during early pregnancy prior to clinical manifestation of the disease. Greater sympathetic output in women with PIH has already been documented in many studies ${ }^{4,5,6}$. 
We applied canonical discriminant analysis to obtain a discriminant model which was able to correctly classify number of cases in one of the two groups. We were able to get high reliability for each test $(92 \% \& 90 \%$ for $\triangle \mathrm{SBP}$ and $\triangle \mathrm{DBP}$ respectively in case of hand grip test; $84 \& 88 \%$ for $\triangle \mathrm{SBP}$ and $\triangle \mathrm{DBP}$ respectively in case of CPT). This means that both these autonomic function tests could very well predict the development of PIH though hand grip test seems to have slightly more predictive accuracy. Sapre ${ }^{13}$ et al have also suggested combination of autonomic function tests to achieve high predictive accuracy.

\section{Conclusion}

Both these simple non-invasive tests i.e. Hand Grip Test \& Cold Pressor Test could be used as important provocative tests in screening women at high risk of developing PIH/PE.

\section{Acknowledgment}

This study was conducted under STS 2008, funded by Indian Council of Medical Research. We deeply acknowledge the same

\section{References}

1. Margaret E, Samuels K, Edmund F et al: Pre pregnancy body mass index, hypertensive disorders of pregnancy and long term maternal mortality. Am J Obstet Gyanecol 2007; 197(5);490.vl-490.c6

2. Mac Kay AP, Berg CJ, Atrash K. Prepregnancy related mortality from preeclampsia and eclampsia. Obstet Gynecol 2001 april;97(4):533-8

3. Khan KS, Wojdyla D, Say L, Gulmezoglu AM, Van LPF: WHO analysis of causes of maternal death: A systematic review. Lancet 2006;367:1066-1074

4. Rang SA, Wolf HA, Montfrans GAvB, Karemaker JMc: Non invasive assessment of autonomic cardiovascular control in normal human pregnancy and pregnancy associated hypertensive disorders: a review. Journal of hypertension 2002;20(11):2111-2119

5. Aireksinen KEJ, Kirkinen P, Takkunen: Autonomic nervous dysfunction in severe preeclampsia. Eur J Obstet Gynecol reprod Biol 1985; 19:269-276

6. Jeltsje S, Cnossen, Joris AM, Vanderpost, Ben WJ, Khalid S, Khan, Catherine A Meads, and Gegen Ter Riet :Prediction of preeclampsia protocol for systemic reviews of test accuracy. BMC Pregnancy and Childbirth 2006, 6:29 doi:10.1186/1471-2393-6-29

7. Greenwood JP, Stoker JB, Walker JJ et al: Sympathetic nerve discharge in normal pregnancy and pregnancy induced hypertension: Journal of Hypertension 1998;16:617-724

8. Dekker GA, Sibai BM: Etiology and pathogenesis of preeclampsia: Current concept. Am J Obstet Gynecol 1998;179:1359

9. Woisetschlager C, Waldenhofer U, Bur A et al: Increased blood pressure response to cold pressor test in pregnant women developing preeclampsia: Journal of hypertension 2000;18(4): 399-403

10. Tomoda S, Kitanaka T, Ogita S, Hidaka A: Prediction of pregnancy induced hypertension by Isometric exercise. Asia Oceania J Obstet Gynaecol 1994;20(3):249-55

11. Degani S, Abinader E, Eibschitz I et al: Isometric exercise test for predicting gestational hypertension. Obstet and Gyane 1985; 65 (5): 652-4

12. Baker PN, Johnson IR: The use of Hand grip in predicting PIH. Eur J Obstet Gyane Reprod Biol.1994;56(3):169-72

13. Sapre S, Sharma N, Joshi V, Sharma P, Sharma AK. Critical evaluation of provocative tests in predicting pre-eclampsia. J Obstet Gynaecol Ind 1996; 46(1): 16-19 